\title{
Vibrational spectra, assignments and normal coordinate analysis for difluorophosphine
}

\author{
VIRGINIA D. DUNNING and ROBERT C. TAYLOR \\ Department of Chemistry. The University of Michigan, Ann Arbor, Ml 48109, U.S.A.
}

(Received 17 July 1978; revised 18 October 1978)

\begin{abstract}
Infrared spectra of the gas and Raman spectra of the liquid and solid forms of $\mathrm{PF}_{2} \mathrm{H}$ and $\mathrm{PF}_{2} \mathrm{D}$ are reported. A normal coordinate analysis based on compliance constants has been carried out on the assigned fundamentals and also on harmonic frequencies estimated by DENNISON's method.
\end{abstract}

\section{INTRODUCTION}

Diffuorophosphine, $\mathrm{PF}_{2} \mathrm{H}$, was first reported and characterized relatively recently by RUDOLPH and PARRY [1]. They reported i.r. frequencies of the gas and solid, suggested tentative assignments and pointed out the marked effect which temperature and physical state had on the stretching frequencies. In particular, they noted that the $\mathbf{P}-\mathrm{H}$ vibration shifted to a higher value upon going from the gas at room temperature to the solid at about $-190^{\circ}$. Results based on studies in argon matrices [2] suggest that strong intermolecular interactions of the dipole-dipole type may exist in the solid, a conclusion which is further substantiated by other physical properties of the substance in condensed phases such as the Trouton constant, NMR chemical shifts, etc. [1] Microwave data [3] show that the molecule is an asymmetric top with a pyramidal structure, the $F-P-F$ and F-P-H angles, respectively, being reported as $99.0^{\circ}$ and $96.3^{\circ}$. Despite the simplicity of the molecule, vibrational assignments are not yet completely certain. No Raman results have been reported because of the tendency of the compound to undergo photodecomposition under normal conditions of excitation.

In the present work, Raman spectra of the liquid and solid have been obtained of the normal and deuterated species and i.r. spectra of the two species have been reexamined in the gaseous state. Assignments have been revised and a normal coordinate analysis has been carried out utilizing compliance constants. Harmonic frequencies estimated by means of DENNISON's method have also been subjected to a normal coordinate analysis.

\section{EXPERIMENTAL}

Difluorophosphine and deutero-difluorophosphine were prepared as outlined in the literature [1] by the reaction of the appropriate phosphine with difluoroiodophosphine in the presence of mercury. The product was purified by careful trap-to-trap distillation on the vacuum line and stored at liquid nitrogen temperatures until use. Vapor pressure and other physical properties agreed with those reported in the literature. Inasmuch as $\mathbf{P F}_{3}$ has bands which interfere with those of $\mathrm{PF}_{2} \mathrm{H}$, particular attention was given in the purification procedures to the removal of $\mathrm{PF}_{3}$.

Since fluorophosphine compounds are very sensitive to impurities, particularly small traces of water, extreme care was taken to keep the vacuum line clean and to dry scrupulously cells and sample containers. Decomposition, when it occurred, could be detected by the formation of a thin yellow film on the container walls which scattered light strongly.

Infrared spectra were obtained using a Beckman IR-12 recording spectrometer which covered the range from 200 to $4000 \mathrm{~cm}^{-1}$. Calibration of the spectrometer with the usual reference substances showed the instrument to be accurate within specifications. The gas spectra were obtained with a standard $10 \mathrm{~cm}$ path length cell with O-ring seals to CsI windows. Solid phase low temperature spectra were obtained using the low temperature cell described by FARRAN [4]. Raman spectra were recorded with a Spex 1401 Ramalog spectrometer using either the $6328 \AA$ line of a He-Ne laser or the $4880 \AA$ line of an argon ion laser as the source of exciting radiation. It was found that the photodecomposition reaction was greatly retarded at low temperatures if the samples had been carefully purified. Consequently, the purified compounds were condensed into $1 \mathrm{~mm}$ diameter capillaries which had been extensively flamed under vacuum prior to use and which were sealed off after partial filling. For spectral examination, the capillaries were inserted into a vacuum jacketed glass tube of approximately $25 \mathrm{~mm}$ outside diameter through which cold nitrogen gas was passing. The temperature of the samples was monitored by a copper-constantan thermocouple and controlled to within $\pm 5^{\circ}$ by adjusting the flow of nitrogen gas. Raman spectra of the solid and liquid phases, including polarization measurements for the latter, were obtained. Estimated uncertainties in frequencies are approximately $1 \mathrm{~cm}^{-1}$.

\section{EXPERIMENTAL RESULTS AND ASSIGNMENTS}

The i.r. spectrum of gaseous $\mathrm{PF}_{2} \mathrm{H}$ is shown in Fig. 1 and the Raman spectrum of the solid is displayed in Fig. 2. Experimental frequencies for $\mathrm{PF}_{2} \mathrm{H}$ are tabulated in Table 1 and those for the deuterated species are given in Table 2 . The molecule has six fundamental frequencies, four totally symmetric $\left(a^{\prime}\right)$ and two asymmetric to the 


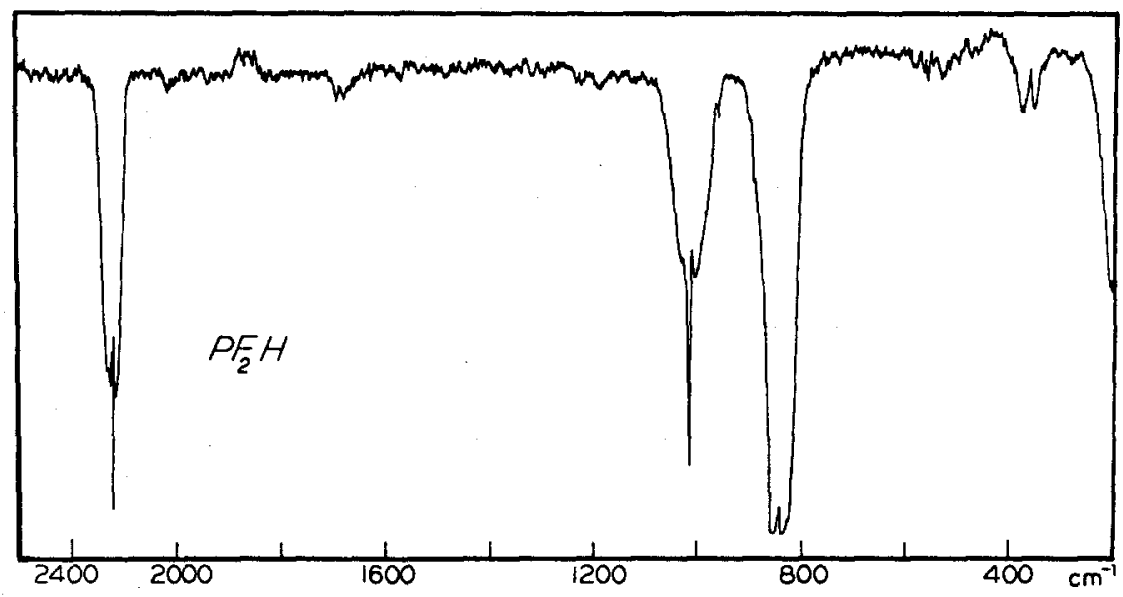

Fig. 1. Infrared spectrum of gaseous $\mathrm{PF}_{2} \mathrm{H}$.

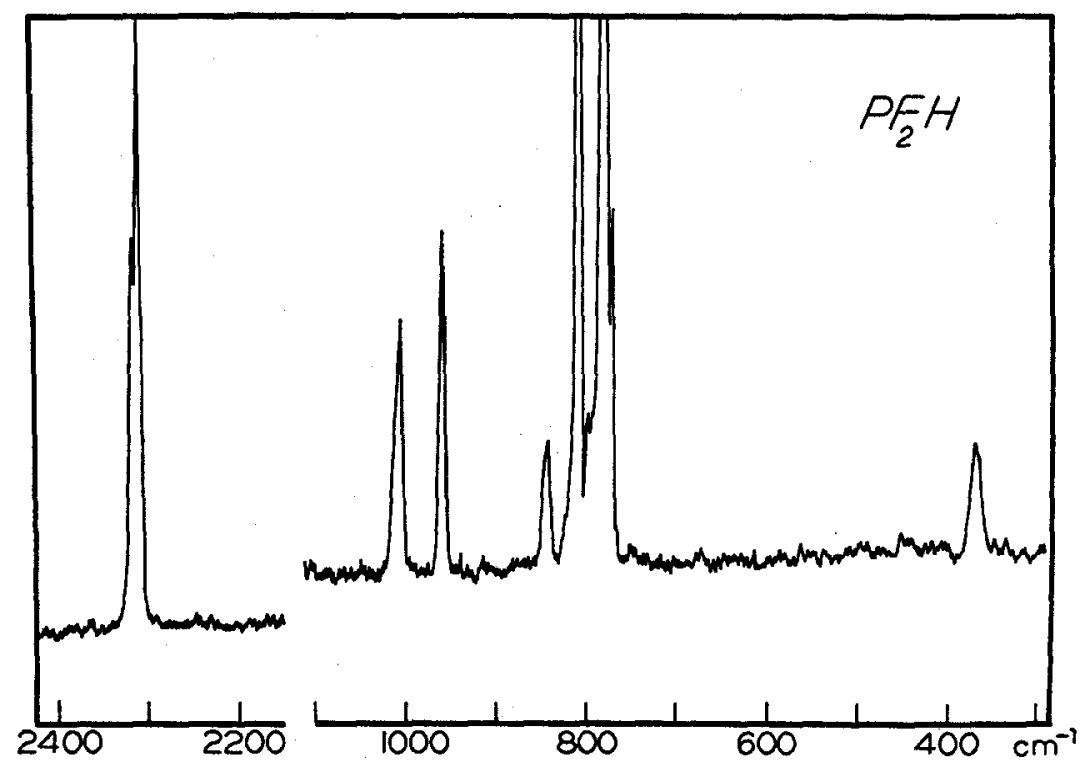

Fig. 2. Raman spectrum of solid $\mathrm{PF}_{2} \mathrm{H}$ at $-150^{\circ} \mathrm{C}$.

plane of symmetry $\left(a^{\eta}\right)$. All frequencies are active in both i.r. and Raman spectra and the normal modes can be described to a good approximation in terms of simple bond stretching and bending motions. The two points of uncertainty with respect to assignments involve the $a^{\prime}$ and $a^{\prime \prime}$ $\mathrm{H}-\mathrm{P}-\mathrm{F}$ deformations and $\mathrm{P}-\mathrm{F}$ stretches. RUDOLPH and PARRY [1] observed two maxima at 1016 and $1008 \mathrm{~cm}^{-1}$ which they assigned respectively to the $a^{\prime}$ and $a^{\prime \prime} \mathrm{H}-\mathrm{P}-\mathrm{F}$ deformations. However, the Raman spectra show there is only one fundamental in this vicinity but that there is a strong depolarized band at $960 \mathrm{~cm}^{-1}$ which has a very weak counterpart in the i.r. This must be the asymmetric deformation, $\nu_{5}$. The three i.r. maxima at 1008,1016 and $1030 \mathrm{~cm}^{-1}$ are therefore assigned to $P, Q$, and $R$ branches of $\nu_{2}$. Upon deuteration, the two deformation frequencies shift to 706 and
$762 \mathrm{~cm}^{-1}$, respectively, the $a^{\prime \prime}$ deformation remaining quite weak in the i.r. All deformation modes appeared relatively insensitive to temperature and phase.

For the P-F stretching frequencies, RUDOLPH and PARRY [1] assigned the $a^{\prime}$ and $a^{\prime \prime}$ modes, respectively, to bands at 851 and $838 \mathrm{~cm}^{-1}$. However, as can be seen in Fig. 1, the bands in tr:s region are quite intense, the two fundamental. overlap strongly and assignments are not immediately apparent. In the case of $\mathbf{P F}_{3}$, it appears well established $[5,6]$ that the symmetric $\left(a_{1}\right)$ mode occurs at a higher frequency than the degenerate (e) mode. Furthermore, the $e$ mode in $\mathrm{PF}_{3}$ is somewhat more sensitive to change in state than the $a_{1}$ mode, the shift from gas to solid at $-180^{\circ}$ being $50 \mathrm{~cm}^{-1}$; the shift of the $a_{1}$ band is $35 \mathrm{~cm}^{-1}[6,7]$. We believe that the $a^{\prime}$ and $a^{\prime \prime} \mathrm{P}-\mathrm{F}$ 
Table 1. Observed i.r. and Raman frequencies of $\mathrm{PF}_{2} \mathrm{H}$

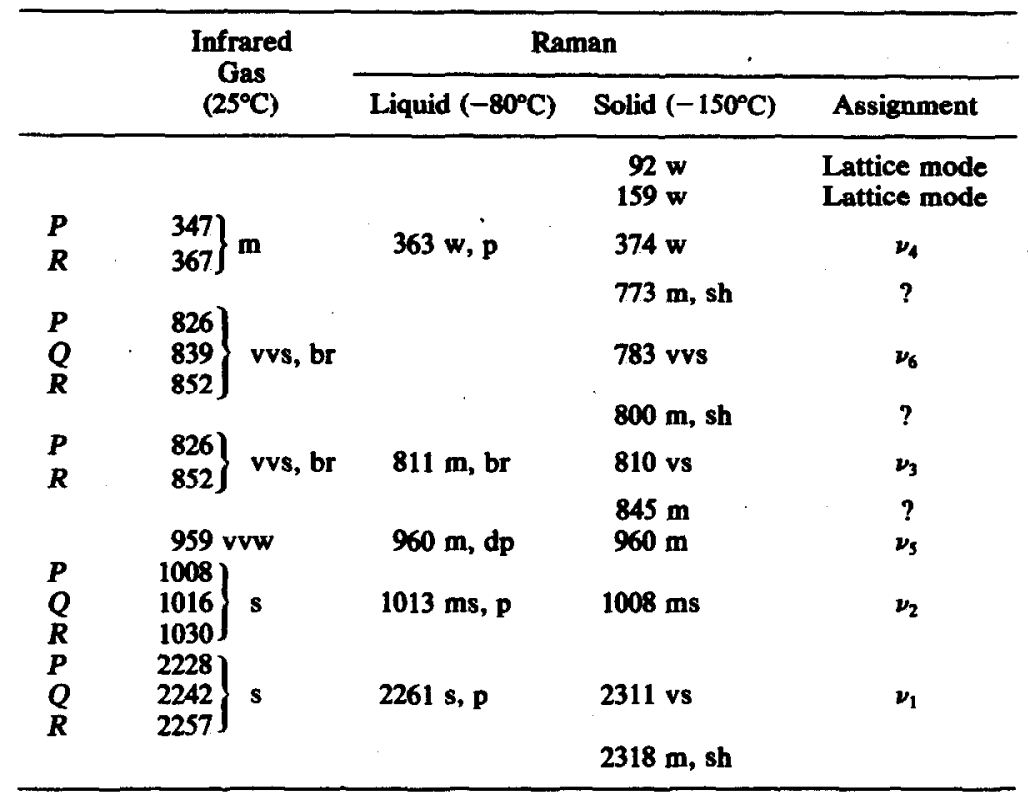

Notation used: $s=$ strong; $m=$ medium; $w=$ weak; $v=$ very; $s h=$ shoulder; br = broad; $p=$ polarized; $d p=$ depolarized.

Table 2. Observed i.r. and Raman frequencies of $\mathbf{P F}_{2} D$

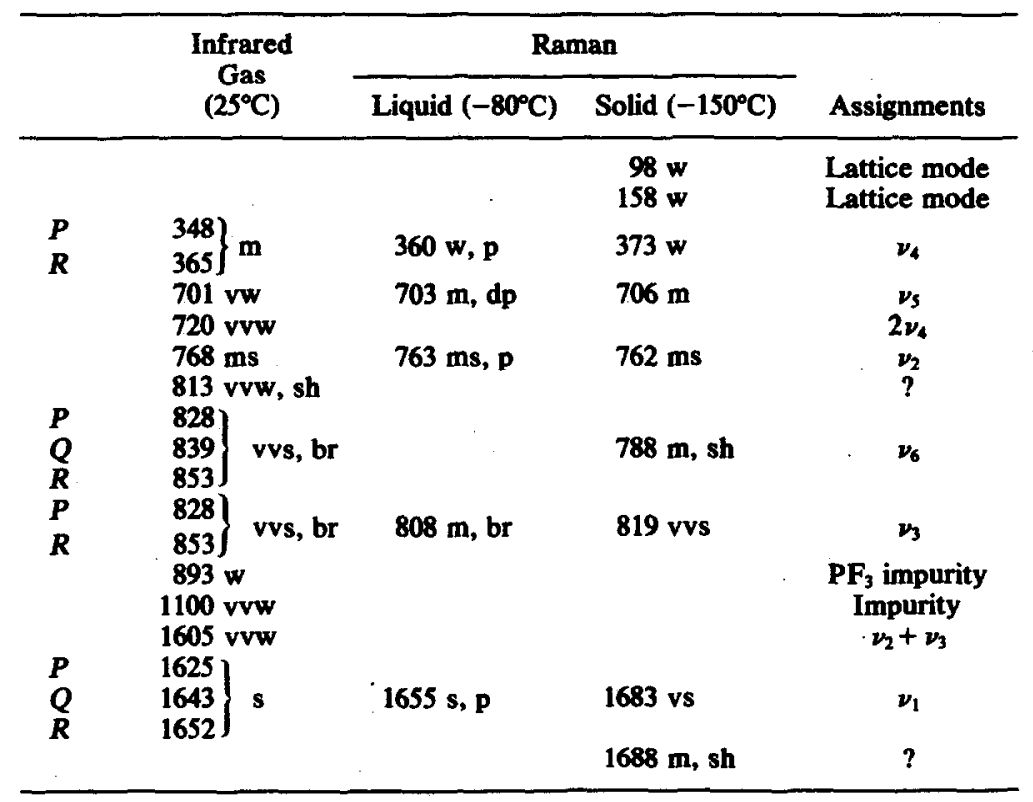

stretching frequencies are accidentally degenerate in the gas spectrum but shift by different amounts on going to the solid spectrum, the two shifts being 29 and $56 \mathrm{~cm}^{-1}$. In the Raman spectrum of the liquid, the separation is not complete and the two bands overlap. However, the highly frequency side of the composite band clearly was polarized which, together with the assumption that the shifts are similar to those of $\mathrm{PF}_{3}$, provides evidence that the $810 \mathrm{~cm}^{-1}$ band of the solid is the $a^{\prime}$ mode and the $783 \mathrm{~cm}^{-1}$ band is the $a^{\prime \prime}$ mode.
The P-F stretching region in the Raman spectrum of the solid was unexpectedly complex and exhibited three distinct maxima in addition to the two fundamentals. No satisfactory explanation for this complexity is apparent although the $P-F$ bonds seem to be particularly sensitive to the known strong intermolecular forces. In this respect it is of interest to note that even in an argon matrix, $P-F$ fundamentals not attributed to aggregate species were reported to be split [2].

As was reported previously, the position of the 
$\mathrm{P}-\mathrm{H}$ stretch was found to be markedly affected by temperature and phase although this region was not complex as was the $P-F$ stretching region. The maximum of the $\mathrm{P}-\mathrm{H}$ stretch shifted approximately $70 \mathrm{~cm}^{-1}$ to higher frequency in the solid compared to the gas, a direction which is opposite to that usually found when hydrogen bonding is present. Apparently, this type of interaction in the solid does not exist. The revised

Table 3. Symmetry coordinates for $\mathrm{PF}_{2} \mathrm{H}$

\begin{tabular}{ll}
\hline$A^{\prime}$ Class & \\
$S_{1}=\Delta d$ & P-H stretch \\
$S_{2}=2^{-1 / 2}\left(\Delta \alpha_{1}+\Delta \alpha_{2}\right)$ & HPF deformation \\
$S_{3}=2^{-1 / 2}\left(\Delta r_{1}+\Delta r_{2}\right)$ & P-F stretch \\
$S_{4}=\Delta \beta$ & PF $F_{2}$ deformation \\
$A^{\prime \prime}$ Class & \\
$S_{5}=2^{-1 / 2}\left(\Delta \alpha_{1}-\Delta \alpha_{2}\right)$ & HPF deformation \\
$S_{6}=2^{-1 / 2}\left(\Delta r_{1}-\Delta r_{2}\right)$ & P-F stretch \\
\hline
\end{tabular}

assignments of the fundamentals are listed in Table 4. For the $A^{\prime}$ class, the theoretical TellerRedlich product rule ratio is 1.860 which can be compared with the experimental value of 1.812 ; corresponding values for the $A^{\prime \prime}$ class are 1.376 and 1.367 , respectively.

\section{VIBRATIONAL ANALYSIS AND DISCUSSION}

Symmetry coordinates and the corresponding numbering scheme for the fundamentals are listed in Table 3. Harmonic frequencies were estimated by DENNISON's method [9] assuming that the anharmonicity coefficient, $x_{i j}$, is the same for all frequencies of a given class. The correction for anharmonicity in the $j$ th frequency is thus given by $\Delta \nu_{i}^{i}=x_{i} \omega_{f} \simeq x_{i} \nu_{l}$ where the index, $i$, refers to the symmetry class. The harmonic frequencies so derived are listed in Table 4.

Table 4. Observed fundamentals and estimated harmonic frequencies for $\mathrm{PF}_{2} \mathrm{H}$ and $\mathrm{PF}_{2} \mathrm{D}$, and differences from calculated values (in $\mathrm{cm}^{-1}$ )

\begin{tabular}{|c|c|c|c|c|c|c|c|c|}
\hline & \multicolumn{4}{|c|}{$\mathrm{PF}_{2} \mathrm{H}$} & \multicolumn{4}{|c|}{$\mathbf{P F}_{2} \mathbf{D}$} \\
\hline & $\nu_{i}$ & $\Delta$ & $\omega_{i}$ & $\Delta$ & $\nu_{i}$ & $\Delta$ & $\omega_{i}$ & $\Delta$ \\
\hline$\nu_{1}$ & 2242 & -16.5 & 2398 & 1.1 & 1642 & 11.4 & 1727 & -0.8 \\
\hline$\nu_{2}$ & 1016.5 & -7.1 & 1049 & -0.5 & 768 & 5.0 & 784 & 0.3 \\
\hline$\nu_{3}$ & 839 & -0.2 & 860 & -0.1 & 839 & -0.3 & 860 & 0.1 \\
\hline$\nu_{4}$ & 357 & 0.3 & 360.5 & 0.0 & 356 & -0.3 & 360 & 0.0 \\
\hline$\nu_{5}$ & 959 & -3.2 & 979.2 & 0.0 & 701.5 & 2.1 & 711.7 & 0.0 \\
\hline$\nu_{6}$ & 839 & 0.0 & 854.4 & 0.0 & 839 & 0.0 & 854.4 & 0.0 \\
\hline
\end{tabular}

$\Delta=$ Experimental - calculated.

Table 5. Symmetry and valence force and compliance constants for $\mathbf{P F}_{2} \mathbf{H}$

\begin{tabular}{|c|c|c|c|c|}
\hline \multirow[b]{2}{*}{ Index } & \multicolumn{2}{|c|}{ Observed frequencies } & \multicolumn{2}{|c|}{ Harmonic frequencies } \\
\hline & $\begin{array}{c}\text { Compliance } \\
\text { constants }\end{array}$ & $\begin{array}{c}\text { Force } \\
\text { constants }\end{array}$ & $\begin{array}{l}\text { Compliance } \\
\text { constants }\end{array}$ & $\begin{array}{c}\text { Force } \\
\text { constants }\end{array}$ \\
\hline \multicolumn{5}{|c|}{ Symmetry constants } \\
\hline 1,1 & 0.354 & 2.891 & 0.309 & 3.275 \\
\hline 2,2 & 0.765 & 1.356 & 0.730 & 1.403 \\
\hline 3,3 & 0.200 & 5.077 & 0.190 & 5.323 \\
\hline 4,4 & 0.790 & 1.265 & 0.775 & 1.290 \\
\hline 5,5 & 1.156 & 0.882 & 1.116 & 0.915 \\
\hline 6,6 & 0.217 & 4.699 & 0.209 & 4.881 \\
\hline 1,2 & 0.078 & -0.301 & 0.051 & -0.230 \\
\hline 1,3 & - & -0.073 & - & -0.050 \\
\hline 2,3 & -0.048 & 0.328 & -0.042 & 0.308 \\
\hline 5,6 & 0.072 & -0.284 & 0.070 & -0.307 \\
\hline \multicolumn{5}{|c|}{ Valence constants } \\
\hline$r(P-F)$ & 0.209 & 4.888 & 0.200 & 5.102 \\
\hline$d(\mathrm{P}-\mathrm{H})$ & 0.354 & 2.891 & 0.354 & 3.275 \\
\hline$\alpha(\mathrm{H}-\mathrm{P}-\mathrm{F})$ & 0.961 & 1.119 & 0.923 & 1.159 \\
\hline $\boldsymbol{\beta}(\mathbf{F}-\mathbf{P}-\mathbf{F})$ & 0.790 & 1.265 & 0.775 & 1.290 \\
\hline$r r$ & -0.009 & 0.189 & -0.010 & 0.221 \\
\hline$r \alpha$ & 0.012 & 0.022 & 0.014 & 0.001 \\
\hline$r \alpha^{\prime}$ & -0.060 & 0.306 & -0.056 & 0.308 \\
\hline$d \alpha$ & 0.055 & -0.213 & 0.036 & -0.163 \\
\hline$\alpha \alpha$ & -0.196 & 0.237 & -0.193 & 0.244 \\
\hline
\end{tabular}

Units: stretch $=\mathrm{mdyn} / \AA \AA$; stretch - bend $=\mathrm{mdyn} ;$ bend $=\mathrm{mdyn}-\AA$. 
Table 6. Thermodynamic properties of $\mathrm{PF}_{2} \mathrm{H}$

\begin{tabular}{|c|c|c|c|c|}
\hline$\underset{{ }^{\circ} \mathbf{K}}{\mathbf{T}}$ & $C_{p} / R$ & $\begin{array}{c}\left(H^{\circ}-H_{0}^{\circ}\right) / \\
R T\end{array}$ & $\begin{array}{c}-\left(G^{\circ}-G_{0}^{\circ}\right) / \\
R T\end{array}$ & $S / R$ \\
\hline 273.15 & 5.536 & 4.504 & 26.821 & 31.325 \\
\hline 298.15 & 5.775 & 4.600 & 27.219 & 31.820 \\
\hline 300 & 5.792 & 4.608 & 27.248 & 31.856 \\
\hline 350 & 6.243 & 4.810 & 27.973 & 32.783 \\
\hline 400 & 6.645 & 5.014 & 28.629 & 33.643 \\
\hline 450 & 6.997 & 5.216 & 29.231 & 34.447 \\
\hline 500 & 7.302 & 5.409 & 29.791 & 35.200 \\
\hline 550 & 7.567 & 5.594 & 30.315 & 35.909 \\
\hline 600 & 7.797 & 5.768 & 30.809 & 36.577 \\
\hline 650 & 7.999 & 5.932 & 31.278 & 37.210 \\
\hline 700 & 8.177 & 6.086 & 31.723 & 37.810 \\
\hline 750 & 8.334 & 6.231 & 32.148 & 38.379 \\
\hline 800 & 8.473 & 6.367 & 32.554 & 38.921 \\
\hline 850 & 8.597 & 6.494 & 32.944 & 39.438 \\
\hline 900 & 8.707 & 6.614 & 33.319 & 39.933 \\
\hline 950 & 8.806 & 6.727 & 33.679 & 40.406 \\
\hline 1000 & 8.895 & 6.833 & 34.027 & 40.860 \\
\hline
\end{tabular}

The normal coordinate analysis was carried out using a standard iterative least squares program to fit both experimental and harmonic frequencies with a potential function expressed in terms of compliance constants. Computational details have been described previously [8]. G matrix elements were evaluated using molecular parameters from the microwave work [3]. Only three off-diagonal constants appeared significant in the refinement, two in the $a^{\prime}$ class and one in the $a^{\prime \prime}$ class. The remaining off-diagonal constants were constrained to zero on the basis of exploratory calculations which indicated their magnitude was likely to be quite small. The analysis confirmed the simple description of the normal modes since the potential energy distribution showed virtually no mixing of coordinates. Differences between observed and calculated frequencies are given in Table 4. Symmetry compliance constants and the corresponding force constants obtained by inversion of the compliance matrices are tabulated in Table 5; the derived valence force and compliance constants are given in the same table.

DUNCAN and Mills [10] have determined reliable values for the force constants of $\mathbf{P H}_{3}$ based on isotopic data and coriolis constants. Their $\mathrm{P}-\mathrm{H}$ valence stretching force constant (harmonic) is $3.45 \mathrm{mdyn} / \AA$ which is not significantly different from the value of $3.28 \mathrm{mdyn} / \AA$ in Table 5. The corresponding values for $f_{P H}$ based on experimental (anharmonic) frequencies are $2.97 \mathrm{mdyn} / \AA$ for $\mathrm{PH}_{3}[10]$ and $2.89 \mathrm{mdyn} / \AA$ from Table 5. Although coriolis data were not available in the present work, the closeness of these numbers suggests that the $\mathrm{P}-\mathrm{H}$ bond is very similar in $\mathrm{PH}_{3}$ and $\mathrm{PF}_{2} \mathrm{H}$.

Force constant data in the literature for $\mathrm{PF}_{3}[6,11-13]$ are not entirely consistent and no "best" value seems available for the $P-F$ bond. In the most recent study by CLARK and RIPPON [6], a value of $4.7 \mathrm{mdyn} / \AA$ for the combination $\left(f_{r}-f_{r}\right)$ was obtained; this is very close to the value for $F_{66}$ in Table 5 which represents the same combination. Other comparisons indicate that the $\mathrm{P}-\mathrm{F}$ bonds in $\mathrm{PF}_{2} \mathrm{H}$ are quite similar to those in $\mathrm{PF}_{3}$ and the net conclusion is that the properties of the intermediate molecule represent a logical interpolation from those of the two binary compounds.

Thermodynamic properties based on observed fundamentals and the usual rigid rotor-harmonic oscillator approximation are given in Table 6.

\section{REFERENCES}

[1] R. W. RudOlPh and R. W. PARRY, Inorg. Chem. 4, 1339 (1965).

[2] J. Burdett, L. Hodges, V. Dunning and J. CuRRENT, J. Phys. Chem. 74, 4053 (1970).

[3] R. L. Kuczkowski, I. Am. Chem. Soc. 90, 1705 (1968).

[4] C. F. FARRAN, Dissertation, The University of Michigan (1966).

[5] T. Shmanouch, Tables of Molecular Vibrational Frequencies, Consolidated Vol. I. U.S. Dept. of Commerce, Washington, D.C. (1972).

[6] R. J. H. Clark and D. M. RIPPON, J. Mol. Spectrosc. 52, 58 (1974).

[7] R. J. WYMA, Dissertation, The University of Michigan (1964).

[8] R. C. TAYLOR, R. W. RUdOLPh, R. J. WyMa and V. D. DunNing, J. Raman Spectrosc. 2, 175 (1974).

[9] D. M. Dennison, Rev. Mod. Phys. 12, 175 (1940).

[10] J. L. DunCaN and I. M. MrLls, Spectrochim. Acta 20, 523 (1964).

[11] A. M. MIRRI, F. Scappini and P. G. Favero, Spectrochim. Acta 21, 965 (1965).

[12] I. W. Levin and S. Abramowitz, J. Chem. Phys. 44, 2562 (1966)

[13] A. M. MirRI, J. Chem. Phys. 47, 2823 (1967). 